\title{
Locating and Collating Translated Short Stories of Rabindranath Tagore
}

SWATI DATTA

\begin{abstract}
Rabindranath Tagore (1861-1941) has been a seminal figure of the Indian literary and cultural scene. His vast and versatile literary contribution includes a large number of short stories that are highly reflective of the sociocultural climate of his times and yet convey ideas and feelings that are perennially relevant to a humanitarian society. Interestingly, the translation world testifies to a history of over one hundred years of English translation of Tagore's Bengali short stories. The paper concentrates on this significant body of translated literature and bases itself primarily on the source text of Galpaguchchha, which is a collection of ninety short stories composed by Tagore. The short stories of Rabindranath are a rich source of crosscultural transmission. That the stories have been translated into English for more than a century now and the translation activity in this sphere still continues, is a phenomenon which merits serious reflection. Keeping in view the complexities of linguistic and cultural transference, the paper discusses the location of this translated literature and attempts a collation of various target language texts. This has been done by analyzing the nature of the stories selected for
\end{abstract}

Translation Today Vol. 2 No. 1 Mar. 2005 @ CIIL 2005 
translation together with the probable reasons for the same and by presenting a comparative study of portions of some of the translations to highlight the translational complexities and nuances. The task to locate and collate the translated short stories, which belong to both the pre- and post-independent years of Indian history, naturally takes into account the complications that develop out of colonial and post-colonial situations. Besides, as over the years, the stories have been translated by Indians as well as non-Indians, the issues like what gets translated, who translates, and for wohom, automatically arises in the course of the projection. Finally, the paper endeavours to see beyond the politics of translation and explores the potential of such translated stories in promoting cross-border solidarities - a feeling that is fundamental in realizing an enlightened multicultural world community.

In one of his famous poems in Chitra, Rabindranath Tagore (1861-1941), a seminal figure on the Bengali literary and cultural scene, addressed his future reader:

"Who are you, reader, reading my poems an hundred years hence?"

(Das 1994: 125)

আজি হতে শতবর্ষ পরে

কে তুমি পড়িছ বসি আমার কবিতাখানি..."

(Rabindra Rachanabali 1961a: 531)

Tagore here visualized a reader of a remote future encountering his writings. Interestingly, a consideration of the English translations of Tagore's short stories would at once relate to a readership across temporal as well as spatial distances. Across 
time, because the translation world testifies to a history of over one hundred years of English translations of Tagore's short stories; and, current translations of his stories reflect a twentyfirst century readertranslator's response to a body of literature composed in the latter half of the twentieth centuries. 'Across space' because the English translations of Tagore short stories involve a cross-cultural transmission, that is, a communication beyond the borders of lands, languages, and cultures.

In recent times, particularly in the fields of literary and culture studies, translation activities have received an impetus, and Tagore's short stories have continued to attract attention. Rabindranath has been a leading exponent of the Bengali short story and has experimented with many styles and techniques. His stories are highly reflective of the sociocultural climate of his times and yet convey ideas and feelings that are perennially relevant to a humanitarian society. Naturally, his work has attracted and influenced contemporary as well as later writers, thinkers, and translators, both at home and abroad. Viewed in this context, English translations of Tagore's short stories offer interesting avenues for critical enquiries. Accordingly, this paper concentrates primarily on the English translations of the short stories of Tagore's Galpaguchchha (Rabindra Rachanabali 1961b), ${ }^{1}$ which literally means a bunch of stories, is a collection of ninety Bengali short stories. This apart, Tagore's Lipika, Se, Tin Sangi, and Galpasalpa together comprise sixty-three stories. However, for the purpose of providing a convenient framework for discussion, Galpaguchchha, which contains the largest number of Tagore's short stories, has been chosen.

\section{Locating the Translated Stories}

English translations of Tagore's short stories belong both to the pre- and post-independent years of Indian history. An attempt to 
locate and collate the translated stories should therefore take into account the complications that develop out of colonial and postcolonial situations. Besides, over the years, the stories have been translated by various hands - Indians as well as nonindians. Another striking feature is that Tagore himself has translated a few of his own stories into English. Hence, issues like what gets translated, who translates, and for whom automatically assume significance.

Tagore has been an abiding influence on modern life and thought. Thematically and stylistically, his short stories are of a wide range and variety. However, translations of his short stories, which appeared in various publications, reveal that certain types of stories have dominated the selection for translation. First, the stories that depict elemental human feelings and predominantly explore human experiences and situations have found projection in many volumes of translations. In this group are included stories like 'Postmaster', 'Khokababur Pratyabartan', 'Kabuliwala', 'Chhuti', 'Subha', 'Samapti', 'Apad' and 'Atithi'. Noticeably, all these stories possess a transnational appeal.

Secondly, there has been a consistent leaning for translations of stories with supernatural overtones. Translations - whether new or reprints or revised versions - of 'Kankal', 'Nishithe', 'Kshudita Pashan', 'Manihara', 'Mastermashai', have recurred in several publications. In fact, the first Macmillan publication of the English translations of Tagore's short stories - Hungry stones and other Stories, published in 1916, derives its name from the title of the English rendering of 'Kshudita Pashan'. Understandably, the bygone Persian setting in the story has appealed to the Western fascination for a fabulous oriental ambience, and the story has appeared and reappeared in translation.

Thirdly, the published translations of Tagore's stories in preindependent India show a preference in selection for politically 'safe' 
and 'non-controversial' stories. Translations of 'Megh o Raudra' and 'Namanjur Galpa', which reflected on colonial domination, did not appear in any of the volumes published before Indian Independence. Yet none other than Edward John Thompson, a liberal British advocate of Indian culture, attempted a translation of 'Megh o Raudra' well before 1947. E.P. Thompson notes that in May 1920 Edward Thompson "wrote to Tagore sending a fragment of 'Cloud and Sunlight' ('Megh O Raudra')". He even mentions that Tagore appears to have informed Macmillan that the story could be included in the next collection. But the "manuscript still remains among his papers and it was not (I think) ever published" (Thompson 1993: 2324). Mary Lago in her biography of Edward John Thompson, titled, "India's Prisoner", also observes, "Edward translated a long story, apparently 'Megh o raudra' (Cloud and sun)" (Lago 2001: 100).

Finally, some of the stories selected for translation are forceful expositions of women's issues although many of them are not to be seen in any of the early volumes of translations. Possibly, trends in feminist developments influenced incorporations of such stories in English translations: renderings of "Denapaona', 'Nashtanir', 'Haimanti', Strir Patra', 'Aparichita', 'Paila Number', are to be found in selections published after 1960.

Having seen some of the features characterising the selection of Tagore's short stories for translation into English, we may turn to the translators that have been engaged in this field. These translators have been both Indians and foreigners, translating during and after the lifetime of the author, and also before and after Indian Independence. Naturally, this body of translated literature encompasses attempts by allied as well as alien minds, the insiders' as well as the outsiders' versions of the original stories. It has indeed been an impressive case of readings by the home and the world. 
Indian translators of Tagore's short stories include eminent persons such as Rajani Ranjan Sen, Panna Lal Basu, Prabhat Kumar Mukherji, Surendranath Tagore, Indira Devi Chaudhurani, Jadunath Sarkar, Amiya Chakravarty, Somnath Maitra, Sujit Mukherjee, Sukanta Chaudhuri, Ranjita Basu, and others. However, recent translations record the efforts of translators of Indian origin Krishna Dutta and Kalpana Bardhan - living abroad and turning back to literature at home. Some of the non-Indian translators are Edward Thompson, W.W. Pearson, C.F. Andrews, Mary Lago, W.W. Pearson, C.F. Andrews, feeling at home in India and working on Indian literature. Not only was this trio considerably acquainted with the source culture but also with the author of the source texts.

Turning to the audience of the translated stories, that is, the target language readers, another interesting and variegated structure comes to view. Obviously and primarily, the target group is a nonBengali readership. It includes non-Bengali Indians and non-Indians. Non-Bengali Indians are somewhat familiar with the Bengali culture due to their proximity to it and the common nationality they share with their Bengali brethren. Non-Indians are comparatively less familiar or completely unfamiliar with the source culture. Among these non-Indians, the people of Great Britain have been in closer interaction with Indian culture because of long years of colonial rule. Nearer home, the people of Bangladesh are pretty much acquainted with the source culture, having once shared the same nationality and still sharing the same source language.

Another feature of the readership of English translations of Tagore's writings is that it surprisingly includes a significant number of Bengali readers too. These Bengali readers are generally of two types. Some of them have had an upbringing outside Bengal and hence are not well conversant in the Bengali language. Others are bilingual, that is, they are comfortable in Bengali and in English, and read the translations out of curiosity or for some specific purposes. 
But their reading usually involves a critical perspective as they are in a position to judge how far the target language text has approximated its source counterpart. Hence, although they do not conventionally belong to the domain of target readers, they are indeed an important and valuable segment of the readership.

\section{Collating the Translated Texts}

Evidently, this varied and cosmopolitan nature of translators and target readers of Tagore's translated short stories casts its impressions on the translations. As it is, translation activities in the field of literature are often ridden by complexities of linguistic and cultural transference. For example, the ailing postmaster in Tagore's eponymous story longs for his mother and elder sister: "তপ্ত ললাটের উপর শাঁখাপরা কোমল হস্তের স্পর্শ মনে পড়ে |". The word 'Shakha' is alien to English culture. To source language readers, however, the contextual use of the word here immediately evokes suggestions of the affectionate, tender, and caressing hands of a married woman - a mother, wife or sister. Debendra Nath Mitter translates the Bengali sentence as: "He remembers the sweet touch of hands, with shell bracelets on, on his fevered brow" (Mitter 1911: 38). The translation in Mashi and Other Stories reads: "He longed to remember the touch on the forehead of soft hands with tinkling bracelets..." (Tagore 1918: 164) Krishna Dutta and Mary Lago have translated the source sentence thus: "He savoured a memory of a soft touch from a bangled hand on a feverish forehead" (Datta \& Lago 1991: 28). William Radice's version is: "He remembered the touch on his forehead of soft hands, conch-shell bangles". (Radice 1994: 44)

Mitter and Radice make some effort to preserve the speciality of 'Shakha' in their respective use of "shell bracelets" and "conch-shell bangles". But the specificity of the bangle is completely 
lost in the other two renderings -"tinkling bracelets" and "bangled hand".

Similarly, when the postmaster tells Ratan that he would soon be leaving, never to return, Ratan is overcome by sadness. Her emotions are conveyed by a stroke of aesthetic ingenuity that is difficult to parallel in translation: "মিটমিট করিয়া প্রদীপ জৃলিতে লাগিল এবং একস্থানে ঘরের জীর্ণ চাল ভেদ করিয়া একটি মাটির সরার উপর টপটপ করিয়া বৃষ্টির জল পড়িতে লাগিল।". While Ratan's tear-drops are echoed in the falling raindrops, the flickering lamp reflects her dampened spirit. Debendra Nath Mitter translates the aforesaid sentence as: "The lamp burnt dimly and pitpat the rain fell on an earthen plate through a chink in the dilapidated thatched roof." (Mitter 1911: 38) In Mashi and Other Stories, the translated sentence reads: "The lamp went dimly burning, and from a leak in one corner of the thatch water dripped steadily into an earthen vessel on the floor beneath it" (Dutta \& Lago 1991: 166). Krishna Dutta and Mary Lago offer the following version: "The lamp flickered, and at one point in the room rain dripped from the decrepit thatched roof into a clay saucer placed on the floor" (Dutta \& Lago 1991: 29). William Radice's translation is: "The lamp flickered weakly; through a hole in the crumbling thatched roof, rain water steadily dripped on to an earthenware dish" (Radice 1994: 45). The onomatopoeic expressions in Bengali, "mitmit" and "toptop", find inadequate representations in all these versions, with Mitter's being the sole attempt to capture the impression of sound in "pitpat". However, the Bengali word, "toptop", echoes both the falling tears and the falling rain while "pitpat" captures the beat of raindrops only.

In 'Shasti', Chandara's decision to embrace the gallows is propelled by a strong feeling of 'abhiman': "এ কী নিদারুণ অভিমান।" This commonly used and extremely evocative Bengali word has 
perplexed translators over the years. Rajani Ranjan Sen in his translation of the story, titled, 'The Sentence', published in Glimpses of Bengal Life, (Sen 1913), steers away from this uncomfortable situation by omitting the sentence. 'Punishment' by Mary Lago and Tarun Gupta, published in The Housewarming and Other Selected Writings, offers the following version: "What a terrible pride this was!" (Chakravarty et.al 1965: 42) Kalpana Bardhan's translation is: "How terribly she was reacting to her hurt feeling..." (Bardhan 1990: 69) Krishna Dutta and Mary Lago write it as: "What unrelenting resentment!" (Dutta \& Lago 1991: 76) William Radice, in his 1991 edition, renders the sentence as, "Such fierce, passionate pride!" and in his 1994 revised-edition as, "Such fierce, disastrous pride!" In both the editions, he appends a footnote: "abhiman: there is no single English word for this emotion. It includes hurt pride, bruised feeling, and rejection by someone we love, Chandara is abhiman incarnate" (Radice 1991: 133). Supriya Chaudhuri's rendering of the said source sentence is: "How terrible was this pride of hers" (Chaudhuri 2000: 118). Radice's footnote is a candid acknowledgement of the absence of an equivalent in target language usage. By what degree an English translation of 'Shasti' stands a chance of missing its mark, becomes evident when one realizes that a sense of 'abhiman' is the quintessence that spins the story.

The choice of an equivalent can be further complicated by the translator's identity and point of view. For instance, William Radice translates "শ্বশুরুনর" in "Jibita o Mrita', as "husband's house" (Radice 1994: 37). In the Indian context, "shashurghar" implies literally and culturally, the father-in-law's house. But Radice naturally reads with an Englishman's eyes and gives the source language expression a western interpretation. Expectedly an Indian translating primarily for a non-Bengali Indian target group would not stray away from the source expression and select "husband's house" because this equivalent does not fit into the conventional 
cultural frame of an Indian family. Another intriguing factor influencing the choice of an equivalent is then the kind of target readership in the translator's mind. When Krishna Dutta and Mary Lago turn the Indian "বটি" into a western "cup" (Dutta \& Lago 1991: 65), in their rendering of 'Madhyabartini,' they actually opt for a target culture-oriented equivalent. Again, in keeping with English etiquette, "খোলাগায়ে" in 'Madhyabartini' becomes "shirtless" (Chakravarty et.al 1965: 45) instead of 'bare-chested' in 'The Girl Between', published in The. Housewarming and Other Selected Writings. In these instances, subtly but surely, the dominant culture tends to prevail over its counterpart. And, the western-oriented equivalents, "husband's house", "cup", "shirtless", become manifestations of the tussle that generally ensues when two cultures that are not at par in power equations, encounter each other in the territory of translation.

\section{Correlating the Author and the Self-translator}

The topic for deliberation also registers the unusual case of the author as the translator of his own stories. Apart from assisting, revising, or partly translating some of his stories, Tagore translated in full three of his stories from Galpaguchchha.These are 'Jayparajay', 'Manbhanjan', and 'Samskar'. These twin roles entwine the self-translator in entanglements that can be variously explained.

Rabindranath Tagore had ventured a Bengali translation of Percy Bysshe Shelley's 'Love's Philosophy' under the title, 'premtattwa'. The original as well as the translated texts of the first stanza are quoted here:

"The fountains mingle with the river

And the rivers with the ocean,

The winds of heaven mix for ever 
With a sweet emotion;

Nothing in the world is single,

All things by a law divine

In one another's being mingle-

Why not I with thine?"

(Palgrave 1954: 185)

“নিঝর মিশিছে তটিনীর সাথে

তটিনী মিশিছে সাগর -' পরে,

পবনের সাথে মিশিছে পবন

চিরস্ুমধুর প্রণয়ভরে!

জগতে কেহই নহিকো একেলা,

সকলি বিধির নিয়মগুণে,

একের সহিত মিশিছে অপরে

আমি বা কেন না তোমার সনে ?"

(Sikdar 1998: 40)

This is indeed an instance of Shelley translated at his best. Tagore here makes his translated lines read natural and he shows an astounding faithfulness to the original. Even the suggestion of original ethereality in "The winds of heaven" is retained in "paban" which means 'wind' or 'air' while. also referring to 'the wind-god' (comparable with the Latin Aeolus and the Greek Aiolos - god of the winds).

What could then possibly have happened to Tagore when he translated his own Bengali writings into English? For, in his selftranslations, the source text often finds a simplified and generalized rendering; and, this is evident in his English translations of his own 
stories too. In fact, the laden implications of the titles, 'Jayparajay' and 'Manbhanjan', have been reduced to only 'The Victory' and a name, 'Giribala', respectively.

In his self-translations, Tagore seems to be more interested in communicating ideas. So, at places, he omits portions of the original or condenses drastically the source language text to a brief and bland expression. For example, of Giribala's elaboration to revenge herself upon her husband in 'Manbhanjan', all that finds representation in translation is: "...prayed in her mind that a day might come when she might have an opportunity to spurn him away with her contempt" (Tagore 1917: 503) The Bengali counterpart however reads: “সে জর্জরিত চিত্তে মনে করিল, যদি কখনো এমন দিন আসে যে, তাহার স্বামী তাহার রূপে আকৃষ্ট হইয়া দগ্ধপক্ষ পতঙ্গের মতো তাহার পদতলে আসিয়া পড়ে, এবহ जে আপন চরণনখরের প্রান্ত হইতে উপেক্ষা বিকীর্ণ করিয়া দিয়া অভিমানভরে চলিয়া যাইতে পারে, তবেই তাহার এই ব্যর্থ রূপ ব্যর্থ যৌঝন সার্থকতা লাভ করিবে।"

Tagore the self-translator also showed an excessive and obsessive concern for his target readers. He nursed a grave anxiety about the degree to which the complex source specificities in his writings could be conveyed across cultures. That is why he sometimes resorted to target-friendly substitutes for source culture specificities. That is why even the simple specificity in “নসস্কার" in 'Jayparajay' gathers a western hue in Tagore's own rendering, "greeted...with a smile and a bow"(Various Writers 1985: 18). In the process of such transformations, the translated stories are shorn of much of their culture specificities and to that extent, the translated texts have become feebler representations of their originals. For example, the humour in re-christening 'Banbihari' as 'Konbihari' in 'Samskar' is lost when Tagore omits in his translation, (Tagore 
1928), the short source sentence: "আমি তার নাম দিয়েছি কোণবিহারী" 'Banbihari' implies roving in forests and groves, especially for pleasure It also bears references to Krishna, who used to rove in groves, playing his flute. In fact, 'bihari' means sporting, frolicking, dallying, promenading, or even indulging in amorous frolics, while 'kon' means an angle or a corner. And the humorous implication of the source sentence is that Banbihari, contrary to his name, is fond of sedentary discussions.

Finally, Tagore was illimitably freer in his creative and imaginative strokes while composing the original stories in his mother tongue than he was while translating them into a language of foreign origin. And in his letters, he has often expressed this apprehension of using English to full advantage. In all this, however, it has to be conceded that Tagore could generously take liberties with the originals because they were his own compositions. Probably, he even assumed that he had the right to take liberties. And so he could afford to forego the translator's fealty to the source text with a note of nonchalance.

\section{Translation - A Transnational Perspective}

Evidently, English translations of Tagore's short stories, like all cross-cultural translations, involve a translocation, a communication across languages and cultures. The source text, rooted in its own culture, is conveyed through another linguistic medium to a target group that generally hails from a different cultural milieu. The Bengali text in English translation thus encounters a new locational context in the target world. Patently or latently, the linguistic and cultural resources of the target language and the kind of target group tend to influence the translated text. Also, a translation ideally aspires to acquire a place in the literary repertoire of the target language, and it has to fulfill the primary 
criterion of readability. At the same time, a translation is invariably judged with reference to its original and this claims that a good translation should make an optimum effort to retain the source specificities. Indeed, most of the translational dilemmas can be traced to those conflicting demands of a shift in location. These complications usually intensify whenever the source and target counterparts do not enjoy equal standing in international power balance. There then arise chances of appropriation or misappropriation of the text and the conquest of the less powerful side by the more dominant one.

However, although propelled by economic logic of taking advantage of the opening of markets, the prevalent tendency all over the world, of regions coming closer and together, has offered tremendous scope for a spurt in translation activities. The European Union (EU), the South Asian Association for Regional Cooperation (SAARC), the Association of South East Asian Nations (ASEAN), and similar other collaborative efforts elsewhere are concrete manifestations of this trend. One of the positive effects of such endeavours is an impetus for interactions amongst different nations and cultures. In the context of these developments, the need for a liberal and transnational outlook of people becomes more and more relevant, and the translators are expected to encash these opportunities.

Fortunately, Tagore's personality, his thoughts and ideas reflected an all encompassing approach that found a crystallized expression in his concept of the Universal Man. Naturally, his writings voiced this vision and this is to be found in many of his short stories too. That is why, in promoting integration amongst nations, his writings have a great potential for translation, and translators are required to respond to this challenge. Possibly, it was Tagore's own transnational attitude that urged him to reach out to an international readership and prompted him to translate his own 
writings into English. After all, English translations enjoy a larger target readership as English has been operating on a global footing.

In this era of interdisciplinary approach and vigorous cooperative ventures amongst nations, translation activity needs to be attended to by a liberated mind that will be, as far as practicable, free from any incriminating influences of major-minor power equations in conveying cultures across national boundaries. The translational forum would thereby ensure mutual respect and appreciation for cultures other than one's own and encourage receptivity and responsiveness to new literatures. In such an atmosphere, source specificities would be conserved as indispensable elements of a world culture and not be mowed down by target language-oriented substitutes of a more dominant culture. It should then be possible to initiate a process of reterritorialisation and relocation by which people will view themselves in a wider context without compromising their distinctive identities. This realization of a symbiotic relationship between one's roots and the world outside would provide a positive perspective for translation as a subject of study as well as an area of operation.

A look into the available English translations of Tagore's short stories reveals that Jatindra M. Bagchi's rendering of the Bengali 'Subha', which appeared in the 16 September 1901 issue of New India: A Weekly Record and Review of Modern Thought \& Life, is the first published English translation. However, a year before this, an attempt at translating Tagore's short stories into English had already been made. Three translated stories were a part of his debut, but only one of these translations appeared in print (Paul 1988: 298) and that too, not until 1912. Yet, in the emerging context of a global village, this maiden translation and its translator acquire a symbolic significance. 


\section{NOTES}

1. All portions quoted in this paper from the Bengali texts of Tagore's short stories bear reference to this publication.

\section{REFERENCES}

Bardhan, Kalpana (Trans.) (1990) 'The Punishment' in Bardhan, Kalpana (ed.) Of Women, Outcastes, Peasants, and Rebels: A Selection of Bengali Short Stories, California: University of California Press.

Chakravarty, Amiya, Mary Lago and Tarun Gupta (Trans). (1965) Punishment in Amiya Chakravarty, (ed.), The Housewarming and Other Selected Writings. New York: The New American Library.

Chakravarty, Amiya, Mary Lago and Tarun Gupta (Trans). (1965) The Girl Between in Amiya Chakravarty, (ed.), The Housewarming and Other Selected Writings. New York: The New American Library.

Das, Sisir Kumar (ed.), (1994) The English Writings of Rabindranath Tagore, Vol. 1. New Delhi: Sahitya Akademi.

Dutta, Krishna and Mary Lago (Trans.), (1991) The Girl Between, in Selected Short Stories of Rabindranath Tagore, London: Macmillan London Ltd. Rpt. 1992, Rupa \& Co., Calcutta.

Dutta, Krishna and Mary Lago (Trans.), (1991) The Postmaster, in Selected Short Stories of Rabindranath Tagore, London: Macmillan London Ltd. Rpt. 1992, Rupa \& Co., Calcutta. 
E.P. Thompson (1993) 'Alien Homage': Edward Thompson and Rabindranath Tagore. Delhi: Oxford University Press.

Lago, Mary (2001) "India's Prisoner": A Biography of Edward John Thompson, 1886-1946, Columbia: University of Missouri Press.

Mitter, Debendra Nath (Trans.) (1911) The Postmaster, Ramananda Chatterjee (ed.), The Modern Review: A Monthly Review and Miscellany, January 1911.

Paul, Prasanta Kumar (1988) Rabijibani, Vol. 4, Calcutta: Ananda Publishers Pvt. Ltd., .

Radice, William (1991) (Trans.) Punishment in Rabindranath Tagore: Selected Short Stories, London: Penguin Books. Rev. ed. (1994).

Radice, William (Trans.) (1994) The Living and the Dead, in Rabindranath Tagore: Selected Short Stories, London: Penguin Books.

Radice, William (Trans.) (1994) The Postmaster, in Rabindranath Tagore: Selected Short Stories, London: Penguin Books.

Sen, Rajani Ranjan (1913) The Sentence, in Glimpses of Bengal Life, Madras: G.A. Natesan \& Co.

Sikdar, Asrukumar (1998) Kabir Anubad, Calcutta: Rabindrabharati Viswavidyalaya.

Supriya Chaudhuri (Trans.) (2000) Punishment, in Sukanta Chaudhuri (Ed.) Rabindranath Tagore: Selected Short Stories. New Delhi: Oxford University Press. 
Tagore, Rabindranath (1917) Giribala Swati Datta (Trans.) Giribala, Ramananda Chatterjee, (ed.) The Modern Review: A Monthly Review and Miscellany. Calcutta, May 1917.

Tagore, Rabindranath (1918) The Postmaster, Mashi and Other Stories London: Macmillan and co. Rpt. 1988, Madras: Macmillan Pocket Tagore Ed., Macmillan India Ltd.

Tagore, Rabindranath (1928) Samskar Tagore, Rabindranath (Trans.) The Patriot, in The Modern Review, July 1928.

Tagore, Rabindranath Rabindra Rachanabali, (posthumous compilation 1961a) Birth Centenary Edition, Vol. 1, Government of West Bengal Publication.

Tagore, Rabindranath Rabindra Rachanabali (posthumous compilation 1961b) Birth Centenary Edition, Vol. 7. Calcutta: Government of West Bengal Publication.

Various Writers (Trans.) (1916) The Victory in Hungry Stones and Other Stories. London: Macmillan. (rpt. 1985) Madras: Macmillan Pocket Tagore Ed., Macmillan India Ltd. 